\title{
Tissue engraftment of hypoxic-preconditioned adipose-derived stem cells improves flap viability
}

\author{
Scott T. Hollenbeck, MD; Annika Senghaas, MD; Issei Komatsu, MD; Ying Zhang, MD; \\ Detlev Erdmann, MD, PhD; Bruce Klitzman, PhD \\ Division of Plastic and Reconstructive Surgery, Duke University Medical Center, Durham, North Carolina
}

\author{
Reprint requests: \\ Dr. S. T. Hollenbeck, Division of Plastic \\ and Reconstructive Surgery, Duke \\ University Medical Center, DUMC 3945, \\ Durham, NC 27710. \\ Tel: +919-681-5079; \\ Fax: +919-681-2670; \\ Email: scott.hollenbeck@duke.edu
}

Manuscript received: December 31, 2011 Accepted in final form: July 19, 2012

DOI:10.1111/j.1524-475X.2012.00854.x

\begin{abstract}
Adipose-derived stem cells (ASCs) have the ability to release multiple growth factors in response to hypoxia. In this study, we investigated the potential of ASCs to prevent tissue ischemia. We found conditioned media from hypoxic ASCs had increased levels of vascular endothelial growth factor (VEGF) and enhanced endothelial cell tubule formation. To investigate the effect of injecting rat ASCs into ischemic flaps, 21 Lewis rats were divided into three groups: control, normal oxygen ASCs $\left(10^{6}\right.$ cells), and hypoxic preconditioned ASCs ( $10^{6}$ cells). At the time of flap elevation, the distal third of the flap was injected with the treatment group. At 7 days post flap elevation, flap viability was significantly improved with injection of hypoxic preconditioned ASCs. Cluster of differentiation-31-positive cells were more abundant along the margins of flaps injected with ASCs. Fluorescent labeled ASCs localized aside blood vessels or throughout the tissue, dependent on oxygen preconditioning status. Next, we evaluated the effect of hypoxic preconditioning on ASC migration and chemotaxis. Hypoxia did not affect ASC migration on scratch assay or chemotaxis to collagen and laminin. Thus, hypoxic preconditioning of injected ASCs improves flap viability likely through the effects of VEGF release. These effects are modest and represent the limitations of cellular and growth factor-induced angiogenesis in the acute setting of ischemia.
\end{abstract}

Interface healing between the flap and recipient wound bed is critical to successful reconstructive surgery. Unfortunately, interface healing must occur at all areas of the flap including points most distal from the flaps primary blood supply. Proper flap healing may be additionally hindered by a nonfavorable recipient wound bed. ${ }^{1}$ Thus, approaches which would improve interface microcirculation might improve healing and overall flap success. Historically, other researchers have sought to address flap ischemia using delivery of soluble growth factors and gene transfer. ${ }^{2-7}$ These results have been somewhat promising in experimental models, yet clinical utility has been limited. Challenges to this approach include the short half-life of exogenous growth factors, unknown effects of viral gene carriers and the unknown optimal timing of growth factor delivery. Based on these hurdles, we wished to take a cell-based approach to address this problem using readily available adipose-derived stem cells (ASCs), considering the concept that autologous cells might produce sustained levels of growth factors and enhance flap angiogenesis.

Mesenchymal stem cells, including ASCs, provide a favorable option for treating ischemic tissue. Specifically, ASCs may migrate in response to basic fibroblast growth factor, ${ }^{8,9}$ secrete vascular endothelial growth factor (VEGF) ${ }^{10,11}$ and hepatocyte growth factor, ${ }^{12}$ and differentiate into endothelial cells. ${ }^{13}$ Moreover, enhancing these effects may not be dependent on genetic modification, but rather environmental conditioning. ASCs are known to react to hypoxic conditions through hypoxia-inducible factor (HIF) signal transduction. ${ }^{14}$ The result of HIF signaling is to promote cell survival in low oxygen conditions and includes metabolic changes and growth factor production. Furthermore, ASCs can be harvested easily, making their clinical utility feasible. Several groups have developed experimental approaches to treat newly infarcted heart tissue with ASCs. In these approaches, the fat-based stem cells have been utilized to enhance vascularization of ischemic tissue through angiogenesis ${ }^{12,15}$ and to differentiate into cardiomyocytes to regain lost pump function. ${ }^{16}$ Based on these observations, we formed the hypothesis that interface wound healing and flap viability may be improved by enhancing neovascularization through local engraftment of hypoxic preconditioned ASCs.

\section{MATERIALS AND METHODS}

\section{HUVEC tubule formation}

Conditioned media were obtained from human ASCs (Zen Bio; Research Triangle Park, Durham, NC) preconditioned for 72 hours in either normal oxygen $(21 \%)$ or hypoxic $(0.5 \%)$ conditions (PROOX Model 110 oxygen controller; Reming Bioinstruments Company, Redfield, NY). Matrigel was placed $(50 \mu \mathrm{L})$ into 96 well plates. A total of 25,000 human umbilical vein endothelial cells ([HUVECs], Clonetics; purchased through Duke Cell Culture Facility) were placed into each well for analysis. After 2-hour incubation, media were removed and replaced with $150 \mu \mathrm{L}$ of control (Dulbecco's modified Eagle medium [DMEM]) or conditioned media. HUVECs were assessed for tubule formation after 72 hours of incubation. Digital images were captured of 
each well and ImageJ software (National Institutes of Health, Bethesda, MD) was used to calculate tubule length in five high-power fields $(100 \times)$.

\section{Rat ASC harvest and evaluation}

All animal studies were performed in conjunction with Duke University Institutional Animal Care and Use Committee. Adult rat (300-350 g) ASCs were isolated from the inguinal fat pad of syngeneic male Lewis rats (Charles River Laboratories, Wilmington, MA) as described previously. ${ }^{17}$ Harvested cells were frozen to allow the same isolation to provide cells for all experiments. The differentiation potential of both fresh isolates and frozen cells was confirmed to ensure that the cells retained their multipotent potential after freezing, data presented elsewhere. ${ }^{17}$

Conditioned media were obtained from rat ASCs following 48 hours of incubation in either normal oxygen $(21 \%)$ or hypoxic $(0.5 \%)$ conditions. At the start of the 48-hour time period, ASCs were placed into minimal media (DMEM with $0.5 \%$ fetal bovine serum). VEGF enzyme-linked immunosorbent assay (ELISA) was performed on conditioned media using the Quantikine assay and protocol (R\&D Systems, Minneapolis, MN) and expressed as means of three separate preparations.

Scratch assays were performed on a confluent monolayer of rat ASCs following 48 hours of incubation in either normal oxygen $(21 \%)$ or hypoxic $(0.5 \%)$ conditions. Following preconditioning; the media was exchanged for fresh minimal media. The cell layer was scratched with a $200 \mu \mathrm{L}$ pipette tip and images were captured at 6, 12, and 24 hours following scratch. The results were expressed as percent of wound repaired with cells migrating from the wound edge.

For chemotaxis assays, rat ASCs were preconditioned in full media for 48 hours in either normal oxygen $(21 \%)$ or hypoxic $(0.5 \%)$ conditions. For the final 24 hours of preconditioning, ASCs were made quiescent by incubation in minimal media. Cells were harvested using $0.05 \%$ trypsinethylenediaminetetraacetic acid (EDTA) and resuspended in minimal. Assays were performed at $37{ }^{\circ} \mathrm{C}$ using a 24-well transwell containing a polycarbonate filter with 8 - $\mu \mathrm{m}$ pores (Poretics, Livermore, CA). Cells were seeded in the upper well at a density of 50,000 cells per well. DMEM alone, VEGF (10 ng/mL, 564-RV; R\&D Systems), or VEGF (10 ng/ $\mathrm{mL})$ plus VEGF-blocking antibody $(0.5 \mu \mathrm{g} / \mathrm{mL}$; AF564; R\&D Systems) was added to the lower well. For chemotaxis assays to extracellular matrix (ECM) proteins, type I collagen (CN) $(20 \mu \mathrm{g} / \mathrm{mL})(\mathrm{C} 5483$; Sigma Aldrich, St. Louis, MO) and laminin (LN) $(20 \mu \mathrm{g} / \mathrm{mL})$ (L4544; Sigma Aldrich) were diluted in minimal media and added to the lower well. After 8 -hour incubation, membranes were removed, fixed in $70 \%$ ethanol at $-20{ }^{\circ} \mathrm{C}$ for 30 minutes, and stained with hematoxylin at room temperature for 30 minutes. The upper side of the membrane was scraped using a cotton swab to remove cells that had attached but not migrated. The membrane was then mounted onto a microscope slide. Chemotaxis was assessed by counting the number of cells that migrated in five independent high-power fields $(200 \times)$.

\section{Surgical protocol}

A well-established model of flap ischemia ${ }^{13}$ was used on 21 Lewis rats. A cranially based $1 \times 3-\mathrm{cm}$ flap was elevated. At the time of elevation with the flap separated from the surrounding wound edges, injection of the distal third of the flap was performed with various experimental groups. A 0.13-mm latex sheet was placed under the flap to prevent wound bed neovasculaization. The flap was sutured in place with 5-0 nylon sutures. The rats were divided into three groups $(n=7$ per group) based on the experimental injection protocol. Control group: $300 \mu \lambda$ of DMEM media was injected into the distal $1 \mathrm{~cm}$ of the flap using a $1-\mathrm{mL}$ syringe with a 25 -gauge needle. Normal oxygen preconditioned ASC group: $300 \mu \lambda$ of DMEM containing $1 \times 10^{6} \mathrm{PKH}-67$ (Sigma-Aldrich)-labeled ASCs preconditioned in normal oxygen (21\%) for 48 hours was injected into the distal $1 \mathrm{~cm}$ of the flap using a $1-\mathrm{mL}$ syringe with a 25 -gauge needle. Hypoxic preconditioned ASC group: $300 \mu \lambda$ of DMEM containing $1 \times 10^{6} \mathrm{PKH}-67-$ labeled ASCs preconditioned in low oxygen $(0.5 \%)$ for 48 hours was injected into the distal $1 \mathrm{~cm}$ of the flap with a 25-gauge needle.

\section{Flap evaluation}

Each rat that underwent skin flap surgery had gross examination performed on postoperative day 7 with digital photography. Digital photographs were evaluated and analyzed by an observer blinded to the assigned treatment group. Adobe Photoshop (Adobe, San Jose, CA) was used to set the pixel value for viable and necrotic tissues. The viability area was determined by subtracting the necrotic area from the total flap surface area. Flap viability was calculated as a percentage of viable surfaces of the total surface area.

\section{Histology}

Following gross evaluation on postoperative day 7, animals were sacrificed and the skin flap with a surrounding 1-cm cuff of normal tissue was harvested and frozen. Cryosections were cut at $5-\mu \mathrm{m}$ thickness and mounted. Tissue sections were pretreated with $3 \%$ hydrogen peroxide to block endogenous peroxidase activity and normal serum to block nonspecific reactions. Sections were incubated at $4{ }^{\circ} \mathrm{C}$ for 12 hours with the primary antibody (mouse-anti rat cluster of differentiation [CD]-31 monoclonal antibody, BD Biosciences Pharmingen, San Diego, CA [1: 100]) and then incubated for 20 minutes at room temperature with secondary antibodies and 3,3'diaminobenzidine reagents from the DAKO Envision Kit (DAKO). Processed slides were viewed in the Duke Microscopy Core facility where digital images were obtained. CD31positive vessels were viewed and staining was subjectively graded as extensive or sparse.

\section{Statistical analysis}

All in vitro assays were performed in triplicate and represent the mean \pm standard deviation. All pairwise differences between two groups were examined using a Student's $t$-test (Microsoft Excel; Microsoft Corp., Redmond, WA) (Figures 2 and 6). The one-way analysis of variance test was used to compare means for the three groups simultaneously (Figures 1 and 3). Post hoc tests were performed to examine the difference between any two groups using the Tukey's honestly significant difference test. A $p<0.05$ was considered significant. 


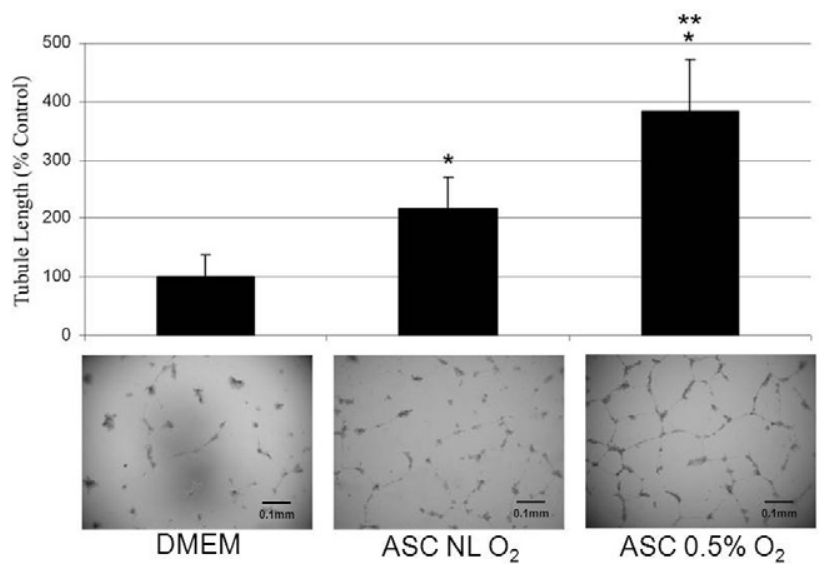

Figure 1. Quantification and representative images of human umbilical vein endothelial cell (HUVEC) tubule formation in response to conditioned media from ASCs. HUVECs cultured in matrigel were exposed to three types of media for 72 hours: DMEM control media, conditioned media from human ASCs cultured in normal oxygen (21\%) for 72 hours, and conditioned media from human ASCs cultured in hypoxic conditions $10.5 \%$ $\mathrm{O}_{2}$ ) for 72 hours. HUVEC tubule formation in conditioned media from both normal oxygen cultured ASCs and hypoxic preconditioned ASCs was significantly greater than DMEM controls (100x). Moreover, HUVEC tubule formation was greatest in conditioned media from hypoxic ASCs. Single asterisks denote statistically significant $(n=3, p<0.05)$ difference from controls. Double asterisks denote statistically significant $(n=3, p<0.05)$ difference between ASC groups.

\section{RESULTS}

\section{Effect of ASC conditioned media on HUVEC tubule formation}

Some researchers believe that ASCs play a role in supporting existing and developing blood vessels. To better understand how hypoxic preconditioning of ASCs might affect the vasculature, we examined the influence of ASC-derived soluble factors on endothelial cell behavior. When cultured in matrigel, HUVEC tubule formation was significantly greater when exposed to conditioned media from both normal oxygen preconditioned ASCs $(218 \pm 54 \%)$ and hypoxic preconditioned ASCs $(382 \pm 90 \%)$ vs. controls $(100 \pm 38 \% ; p<0.05)$ (Figure 1). Moreover, conditioned media from hypoxic ASCs resulted in significantly greater HUVEC tubule formation than conditioned media from normal oxygen preconditioned ASCs $(382 \pm 90 \%$ vs. $218 \pm 54 \% ; p<0.05)$. This indicates that soluble factors (found in conditioned media) from normoxia and hypoxic preconditioned ASCs promote HUVEC tubule formation.

\section{Effect of hypoxic preconditioning on ASC VEGF production}

Based on the observation that hypoxic human ASCs enhance endothelial cell tubule formation through soluble mediators, we wished to determine if this could be applied to a surgical model to improve flap survival. Anticipating a rat model for tissue ischemia, we next wished to verify that rat ASCs produced proangiogenic adipokines, specifically VEGF, in response to hypoxia. Using ELISA, conditioned media from hypoxic $\operatorname{ASCs}\left(0.5 \% \mathrm{O}_{2}\right.$ for 48 hours $)$ were found to have significantly greater concentrations of VEGF than conditioned media from normal oxygen $\left(21 \% \mathrm{O}_{2}\right.$ for 48 hours $)$ controls $\quad(3215 \pm 173.1 \mathrm{pg} / \mathrm{mL} \quad$ vs. $2476 \pm 108 \mathrm{pg} / \mathrm{mL}$; $p<0.05$ ) (Figure 2A).

\section{Effect of VEGF on ASC chemotaxis}

Since ASCs produced VEGF, we wanted to determine whether VEGF could induce ASC chemotaxis in an autocrine manner and influence ASC dispersion following flap delivery. To test this, we performed transwell migration assays on ASCs exposed to minimal media control and VEGF, with and without a blocking antibody to VEGF. ASC migration was significantly increased in response to VEGF $(10 \mathrm{ng} / \mathrm{mL})$
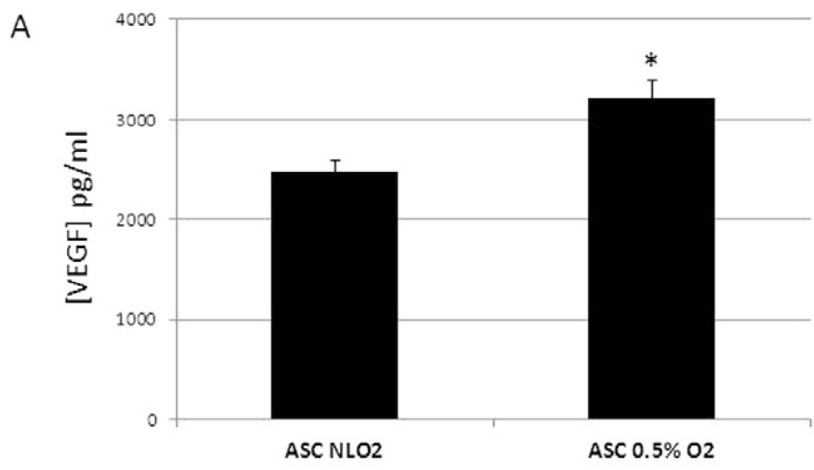

B

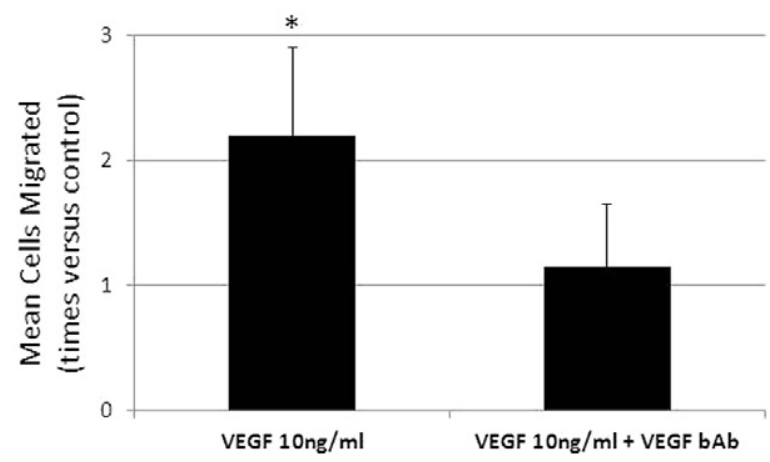

Figure 2. Quantification of ASC VEGF production and chemotaxis. (A) The mean ELISA results for soluble VEGF found in conditioned media are shown. Media from hypoxic preconditioned ASCs (ASC $0.5 \% \mathrm{O}_{2}$ ) contains significantly higher levels of VEGF than media from normal oxygen preconditioned ASC (ASC NL $\mathrm{O}_{2}$ ). Asterisks denote statistically significant $(p<0.05)$ difference $(n=3)$. (B) The mean number of ASCs migrated in response to VEGF is shown relative to controls. VEGF $(10 \mathrm{ng} / \mathrm{mL})$ stimulated ASC chemotaxis significantly greater than control (DMEM) and this effect was reversed with blocking antibodies to VEGF. Asterisks denote statistically significant $(p<0.05)$ difference from controls $(n=3)$. 
A

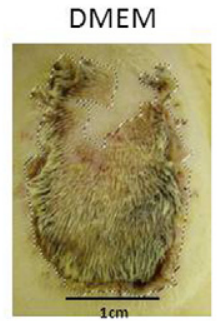

B

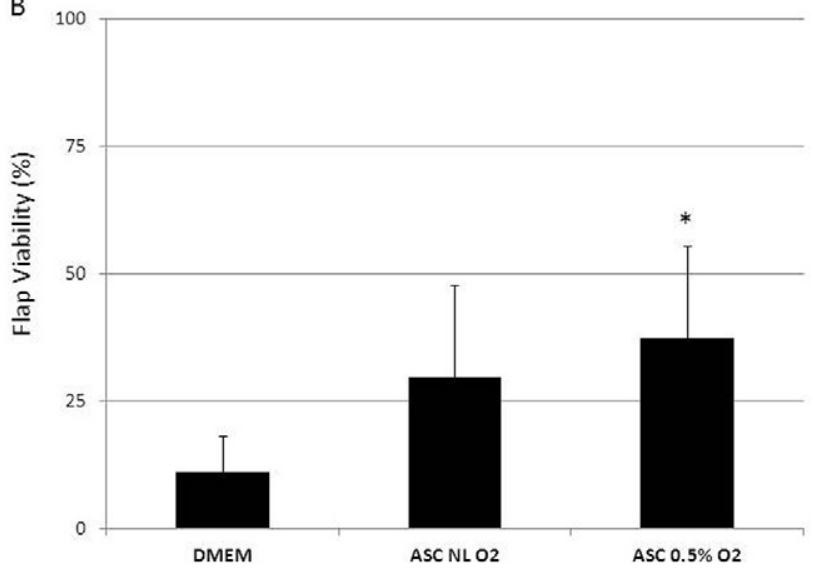

Figure 3. Comparison of flap survival areas. (A) Representative photographs of flaps at postoperative day 7 . The base and cranial portion of the flap are at the upper aspect of the image. (B) The mean flap viability was significantly greater for hypoxic preconditioned ASC engrafted flaps than DMEM controls. Asterisks denote statistically significant $(p<0.05)$ difference from control ( $n=21,3$ groups of 7$)$.

containing minimal media $(2.2 \pm 0.7$ times over control, $p<0.05)$ (Figure 2B). Following the addition of VEGFblocking antibody $(0.5 \mu \mathrm{g} / \mathrm{mL})$, ASC chemotaxis to VEGF was decreased to basal levels $(1.2 \pm 0.5$ times over control; $p>0.05)$. Taken together, these results suggest that ASCs produce VEGF which may act in an autocrine manner to stimulate chemotaxis.

\section{Effect of engrafted ASCs on flap viability}

Based on our initial observations, we wished to determine whether hypoxic preconditioned ASCs could be engrafted into soft tissue flaps to enhance angiogenesis and viability. To test this idea, we used a well-described rat model for flap ischemia. On gross examination at 7 days post flap elevation, the DMEM control groups appeared to have the most distal tip necrosis and proximal flap ischemia (Figure 3). The normal oxygen preconditioned ASC engrafted flaps appeared to have less distal tip necrosis and less proximal flap ischemia. Finally, the hypoxic preconditioned ASC engrafted flaps appeared to have the least distal tip necrosis and proximal flap ischemia. Image analysis confirmed that flaps engrafted with hypoxic preconditioned ASCs had significantly better overall viability (control: $11.2 \pm 7$, normal ASCs: $29.8 \pm 18$, hypoxic ASCs: $37.4 \pm 18 ; p<0.05$ ) (Figure 3 ).

To investigate how ASCs might improve flap viability, we performed immunohistochemistry to assess blood vessel formation at the margins of the flap in the distal third where the ASCs had been injected. CD31 staining showed extensive neovascularization at the distal margins of the ASC injected flaps (Figure 4). In contrast, there was sparse neovascularization seen at the margins of control flaps.

\section{Localization of engrafted ASCs}

As ASC injected flaps had improved margin neovascularization and overall viability, we wanted to determine where within the tissue the ASCs had localized, too, to better understand how this effect may have occurred. While all the samples were injected into the subcutaneous tissue diffusely, we found a unique distribution between the normal oxygen preconditioned ASCs and the hypoxic preconditioned ASCs (Figure 5). Using fluorescence to detect the PKH-67 label, we found the normal oxygen ASCs localized primarily around existing large-caliber blood vessels. In contrast, hypoxic preconditioned ASCs were found diffusely throughout the subcutaneous tissue and even across the flap native skin interface. This indicated that both groups of cells had migrated from the original site of injection in a unique pattern.

\section{Effect of hypoxia on ASC migration and chemotaxis}

Since we had observed a unique migration pattern between the normal oxygen preconditioned ASCs and the hypoxic preconditioned ASCs within our flaps, we wished to determine if hypoxic preconditioning significantly altered ASC migration and chemotaxis. To test this, we performed a scratch assay with normal oxygen and hypoxic preconditioned ASCs. Wound repair rate and extent was similar in both groups (Figure 6A).

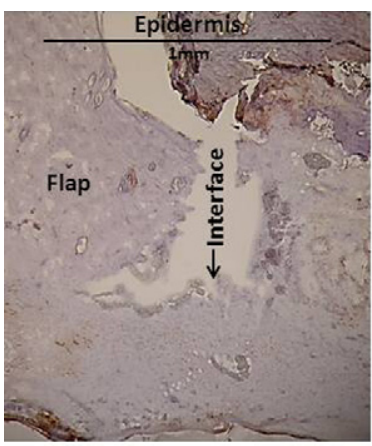

DMEM

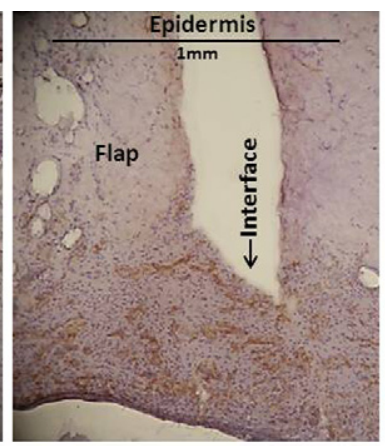

ASC NL $\mathrm{O}_{2}$



ASC $0.5 \% \mathrm{O}_{2}$
Figure 4. Photomicrographs of CD31 immunohistochemistry (20x). The images depict the lateral portion of the distal $1 / 3$ rd of the flap-native skin interface. At the margin of the flap wound interface, CD31 expression was sparse in DMEM control injected flaps. In contrast; at the margin of the flap wound interface, CD31 expression was extensive in both normal oxygen and hypoxic preconditioned ASC injected flaps. 

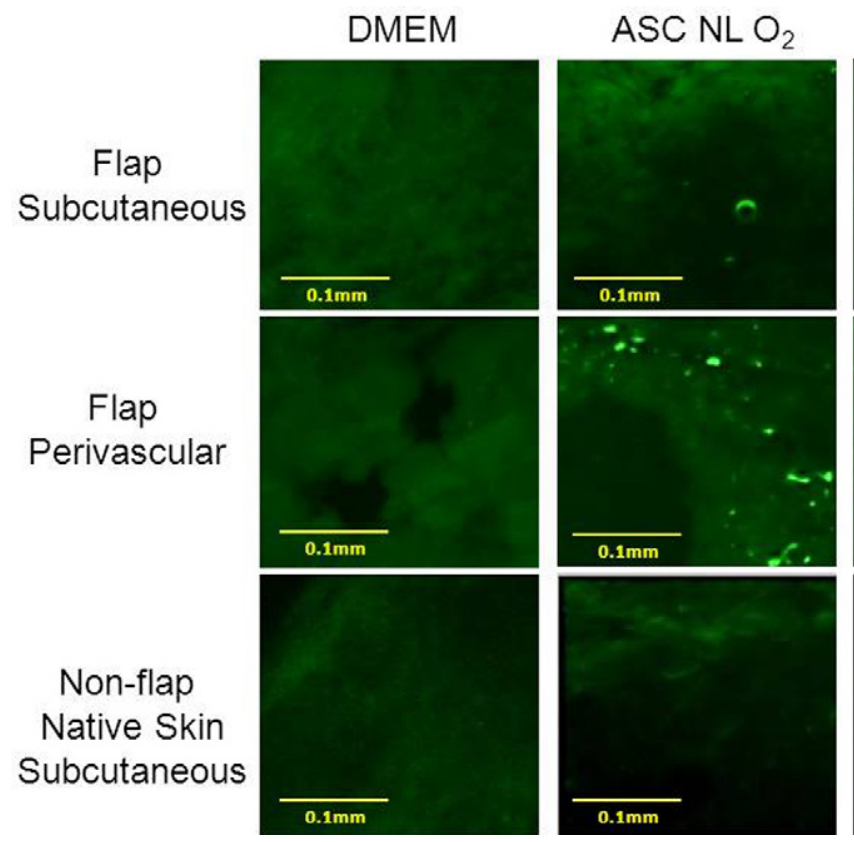

Next, we explored the chemotactic response of the ECM proteins type I CN and $\mathrm{LN}$. Both proteins are known to be important structural components of dermis and subcutaneous tissue as well as chemotactic stimuli to migrating cells. Following preconditioning in normal oxygen and hypoxic conditions, ASC migration in response to $\mathrm{CN}(20 \mu \mathrm{g} / \mathrm{mL})$ was significantly greater than in response to control media $(4 \pm 1.1$ and $6 \pm 0.5$ times, respectively; $p<0.05$ ) or LN (Figure $6 \mathrm{~B}$ ). While not statistically significant, hypoxic preconditioned ASCs showed a stronger migratory response to $\mathrm{CN}$ than normal oxygen preconditioned ASCs. ASC migration to laminin $(20 \mu \mathrm{g} / \mathrm{mL})$ was not significantly different than controls and was not significantly affected by hypoxic preconditioning. These data suggest that hypoxic preconditioning does not adversely alter ASC migration or chemotaxis to ECM proteins found within the dermis and subcutaneous tissues. Thus, distinct patterns of ASC distribution seen within the flap do not appear to solely relate to the preconditioning process.

\section{DISCUSSION}

Traditionally, efforts to improve distal flap margin blood flow have involved surgical delay, a process involving division of a portion of the flaps blood flow. Following surgical delay, the flap becomes dependent on the remaining vasculature which must undergo changes to accommodate the altered blood flow. After a period of 2 to 3 weeks, the altered blood flow within the delayed flap will support the tissue during transfer. It is believed that surgical delay creates an environment of relative hypoxia which stimulates angiogenesis and vessel dilation. ${ }^{18}$ Prior studies have shown that during surgical delay, local tissue at the edge of the primary angiosome is under ischemic conditions and this results in a generalized production of growth factors within the tissue, including VEGF. ${ }^{19}$ As a result, there is increased angiogenesis at these sites combined with dilation of choke vessels, leading to more blood flow to
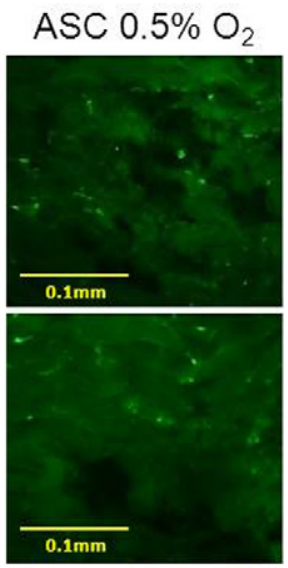

Figure 5. Photomicrographs of $\mathrm{PKH}-$ 67-labeled ASCS under green fluorescence (200x). Within the central and distal portion of the flap, normal oxygen preconditioned ASCs (ASC NL $\mathrm{O}_{2}$ ) are sparsely distributed in the general subcutaneous tissue and densely organized around large blood vessels. Hypoxic preconditioned ASCs (ASC $0.5 \% \mathrm{O}_{2}$ ) are seen distributed in a more diffuse pattern. Across the flap interface within non-flap native tissue, only hypoxic preconditioned ASCs are identified. DMEM injected flaps are shown for comparison. All sections are oriented with epidermis up.

the edge of the flap. Through ex vivo cellular preconditioning, we have tried to achieve a "cellular delay" which theoretically drives the production of proangiogenic factors from mesenchymal stem cells prior to any tissue ischemia.

The effect of hypoxia as a stimulator of angiogenesis is well described. It is known that both endothelial cells and vascular smooth muscle cells possess $\mathrm{O}_{2}$ sensitive enzymes which allow for cellular changes to occur in the setting of hypoxic conditions..$^{20,21} \mathrm{Hu}$ and colleagues demonstrated that hypoxic preconditioned bone marrow-derived mesenchymal stem cells (BM-MSCs) transplanted into infarcted heart tissue could significantly enhance angiogenesis ${ }^{15}$ within the damaged region. Hu noted that these hypoxic preconditioned stem cells had enhanced expression of hypoxia-inducible factor (HIF1-alpha) and multiple angiogenic factors including VEGF. This activity is in line with the role of BM-MSCs, that of support for the stroma and hematopoietic stem cells within the marrow. What has been unclear is the potential angiogenic capacity of tissue-based mesenchymal stem cells-including ASCs. Yet, there is evidence that ASCs are involved in the hypoxia response within tissues. For example, Rehman and others demonstrated that ASCs cultured in hypoxic conditions will up-regulate VEGF secretion fivefold. ${ }^{11}$ While there is a significant amount of evidence that BM-MSCs play a role in tissue repair through angiogenic responses to hypoxia, we have shown in this study that peripheral-based mesenchymal stem cells appear to share a similar capacity.

It is interesting to note that our findings are similar to prior studies in which flaps were supplemented with exogenous VEGF. Overall, we found a $26 \%$ increase in flap viability with hypoxic ASC flap injection. Seify and colleagues, using a rat transverse rectus abdominus myocutaneous flap model, found a $37 \%$ increase in flap viability following injection of intraarterial VEGF. ${ }^{3}$ This compared favorably with surgical delay alone. In another study investigating random skin flaps, Padubidri et al. demonstrated an $18 \%$ increase in flap viability 

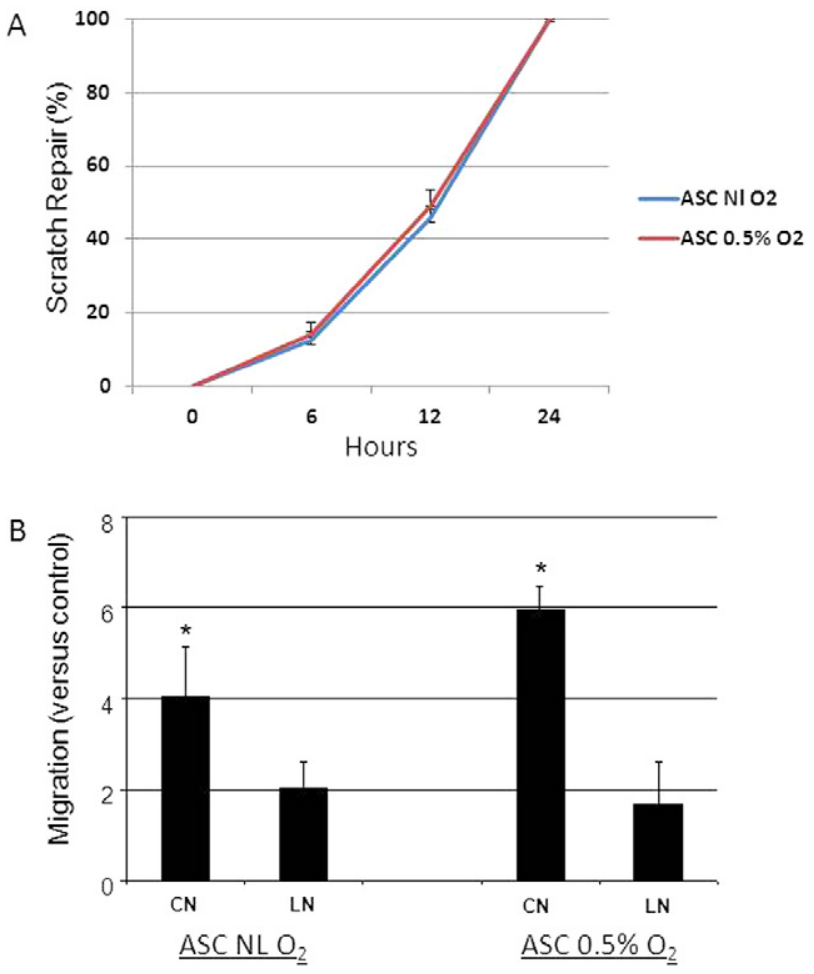

Figure 6. Quantification of ASC migration and ECM chemotaxis. (A) Mean results for scratch assay wound repair with normal and hypoxic ASCs. No significant difference was observed $(n=3)$. (B) Cellular migration through transwell chambers in response to soluble CN $(20 \mu \mathrm{g} / \mathrm{mL})$ and $L N$ $(20 \mu \mathrm{g} / \mathrm{mL})$. Normal oxygen and hypoxic preconditioned ASCs migrated significantly greater to $\mathrm{CN}$ than to DMEM controls or $L N$. Asterisks denote statistically significant $(p<0.05)$ differences from control and $\mathrm{LN}(n=3)$.

following intra-arterial VEGF injection. In both instances, the improvement in flap viability was presumed to be related to increased angiogenesis secondary to increased flap VEGF concentrations. This is consistent with our findings; still, this effect remains insufficient to produce complete flap survival.

Cell migration within the ECM is fundamental to cellular homeostasis and tissue integrity. Likewise, effective use of cellular therapeutics such as those employed in this study will be dictated by an understanding of cell migratory stimuli. The subcutaneous tissue is rich in ECM proteins, including type 1 $\mathrm{CN}$ and $\mathrm{LN} .^{22,23}$ Thus, we wanted to determine how ASCs would respond to ECMs in both normal and hypoxic conditions. Interestingly, migration to type $1 \mathrm{CN}$ and $\mathrm{LN}$ was not adversely affected by hypoxic preconditioning. The next question is whether the engrafted cells will remain at the injection site, or will they migrate to other areas within the tissue. Engraftment of ASCs into the proximal portion of flaps has been shown to be beneficial to flap perfusion. ${ }^{13}$ In those studies, transplanted cells seemed to remain localized at the engraftment site. Ceradini and colleagues demonstrated that local tissue hypoxia up-regulates stromal cell-derived factor-1
(SDF-1) through HIF-1 alpha signaling. ${ }^{24}$ Furthermore, SDF-1 may act as a chemokine for circulating progenitor cells leading to precise localization at the site of hypoxia. This suggests a mechanism for enhanced trafficking of BM-MSCs to ischemic peripheral tissue, thus suggesting a role in tissue repair. As we have demonstrated a strong migratory capacity of ASCs in normal and hypoxic conditions, one may derive that a similar signal might be in place for localizing peripherally based ASCs to hypoxic tissue in preparation for angiogenesis.

Migration of cells through tissue is a well-described process. In this report, we demonstrate a novel finding of ASC migration from the engraftment point within the flap across a wound interface into the surrounding native tissue. This was an unexpected finding in the sense that these cells are migrating from a hypoxic region to a relatively nonhypoxic region. Interestingly, this was observed only in the hypoxic preconditioned ASC group. The normal oxygen preconditioned ASCs appeared to all remain within the flap. This raises the possibility that hypoxic preconditioned stem cells engrafted into hypoxic tissue are responding to a signal from an existing blood vessel lying within nonhypoxic tissue. We might conclude that this localization is consistent with the role of ASCs as vascular pericytes involved in assisting endothelial cells in the angiogenesis process. In this regard, the hypoxic preconditioned ASCs may support endothelial cells, perhaps through VEGF production, as they advance toward the wound edges and the ischemic tissues of the flap. Again, in this regard, we tend to agree with the findings of Amos and colleagues that show that ASCs injected into tissues take on a perivascular morphology and are associated with increased blood vessel density. ${ }^{25}$

Another potential role for ASCs in tissue repair would be that of differentiation into specific cell types needed for regeneration and/or wound healing. Wu et al. has demonstrated that BM-MSCs injected into wounds can both stimulate angiogenesis through VEGF and differentiate into dermal elements including skin appendages. ${ }^{26}$ Still, others have suggested that injected ASCs may become endothelial cells of developing blood vessels. In contrast to findings by Lu et al. we did not see engrafted-labeled ASCs within new blood vessels or skin appendages. ${ }^{13}$ However, as mentioned previously, we did repeatedly see PKH-labeled ASCs associated with existing large-caliber blood vessels, particularly the normal oxygen preconditioned ASCs. Taken together, these findings suggest that the role of ASCs in ischemic tissue may be supportive in nature rather than regenerative, consistent with pericyte lineage.

Overall; this study showed an improvement in flap viability when the flap was injected with preconditioned ASCs. However, this effect was moderate and not sufficient to prevent some degree of flap necrosis and ischemia. This is consistent with multiple other studies in which growth factors are added to a surgical flap to induce angiogenesis. ${ }^{5-8}$ This approach appears to be limited due to the amount of time necessary for new blood vessel formation. ${ }^{27}$ The ischemic tissue undergoes necrosis prior to therapeutic angiogenesis, even in the presence of additional growth factors. As we look at ways to improve interventions on acute ischemia, we feel that it is important to focus on principles described in surgical delay - that of larger vessels dilation and remodeling. ${ }^{28}$ The manipulation of existing vessels is likely to result in a more rapid increase in perfusion to ischemic areas than therapeutic angiogenesis. For this reason, we are currently looking at the 
role of ASCs within periadventitial fat and the potential to enhance vasodilation.

In summary, hypoxic preconditioned ASCs appear to enhance proangiogenic features of endothelial cells through both soluble mediators and direct cell-to-cell interactions. When injected into the distal portion of flaps, hypoxic preconditioned ASCs migrate throughout the flap and across flap margins to the native tissue. This corresponds to improved flap viability and oxygenation. These results suggest that ASCs may act in a supporting role for hypoxia-induced angiogenesis within tissue. Moreover, it may be possible utilize these properties to enhance vascularization of autologous and engineered constructs.

\section{ACKNOWLEDGMENTS}

This project was supported by a Plastic Surgery Education Foundation Basic Science Research Grant.

This work was presented at the 2009 Southeastern Society of Plastic and Reconstructive Surgeons in San Juan, Puerto Rico and the 2009 Plastic Surgery Research Council in Pittsburgh, Pennsylvania.

Conflict of Interest: The authors have no conflicts of interest to report in regard to this manuscript.

\section{REFERENCES}

1. Rudolph R. Complications of surgery for radiotherapy skin damage. Plast Reconstr Surg 1982; 70: 179-85.

2. Padubidri A, Browne E Jr. Effect of vascular endothelial growth factor (VEGF) on survival of random extension of axial pattern skin flaps in the rat. Ann Plast Surg 1996; 37: 604-11.

3. Seify H, Bilkay U, Jones G. Improvement of TRAM flap viability using human VEGF-induced angiogenesis: a comparative study of delay techniques. Plast Reconstr Surg 2003; 112: 1032-9.

4. Seify H, Bulky U, Jones G. Effect of vascular endothelial growth factor-induced angiogenesis on TRAM flap harvesting after abdominoplasty. Plast Reconstr Surg 2003; 111: 1212-16.

5. Taub P, Marmur J, Zhang W, Senderoff D, Urken M, Silver L, Weinberg H. Effect of time on the viability of ischemic skin flaps treated with vascular endothelial growth factor (VEGF) cDNA. $J$ Reconstr Microsurg 1998; 14: 387-90.

6. Zhang F, Fischer K, Komorowska-Timek E, Guo M, Cui D, Dorsett-Martin W, Buncke H, Lineaweaver W. Improvement of skin paddle survival by application of vascular endothelial growth factor in a rat TRAM flap model. Ann Plast Surg 2001; 46: 314-19.

7. Lee WJ, Yun CO, Yun IS, Kim YO, Choi IK, Yun TJ, Rah DK. Augmentation of rat skin flap viability by relaxin-expressing adenovirus. Wound Repair Regen 2011; 19: 709-17.

8. Marra K, DeFail A, Clavijo-Alvarez J, Badylak S, Taieb A, Schipper B, Bennett J, Rubin J. FGF-2 enhances vascularization for adipose tissue engineering. Plast Reconstr Surg 2008; 121: 1153-64.

9. Schmidt A, Ladage D, Schinkothe T, Klausmann U, Ulrichs C, Klinz F, Brixius K, Arnhold S, Desai B, Mehlhorn U, Schwinger RH, Staib P, Addicks K, Bloch W. Basic fibroblast growth factor controls migration in human mesenchymal stem cells. Stem Cells 2006; 24: 1750-58.

10. Nakagami H, Morishita R, Maeda K, Kikuchi Y, Ogihara T, Kaneda Y. Adipose tissue-derived stromal cells as a novel option for regenerative cell therapy. J Atheroscler Thromb 2006; 13: 77-81.

11. Rehman J, Traktuev D, Li J, Merfeld-Clauss S, Temm-Grove C, Bovenkerk J, Pell C, Johnstone B, Considine R, March K. Secretion of angiogenic and antiapoptotic factors by human adipose stromal cells. Circulation 2004; 109: 1292-8.

12. Zhu X, Zhang X, Xu L, Zhong X, Ding Q, Chen Y. Transplantation of adipose-derived stem cells overexpressing hHGF into cardiac tissue. Biochem Biophys Res Commun 2009; 379: 1084 90.

13. Lu F, Mizuno H, Uysal C, Cai X, Ogawa R, Hyakusoku H. Improved viability of random pattern skin flaps through the use of adipose-derived stem cells. Plast Reconstr Surg 2008; 121: $50-8$.

14. Grayson WL, Zhao F, Bunnell B, Ma T. Hypoxia enhances proliferation and tissue formation of human mesenchymal stem cells. Biochem Biophys Res Commun 2007; 358: 948-53.

15. Hu X, Yu S, Fraser J, Lu Z, Ogle M, Wang J, Wei L. Transplantation of hypoxia-preconditioned mesenchymal stem cells improves infarcted heart function via enhanced survival of implanted cells and angiogenesis. J Thorac Cardiovasc Surg 2008; 135: 799-808.

16. Van Dijk A, Niessen H, Zandieh Doulabi B, Visser F, van Milligen F. Differentiation of human adipose-derived stem cells towards cardiomyocytes is facilitated by laminin. Cell Tissue Res 2008; 334: 457-67.

17. Prichard H, Reichert W, Klitzman B. IFATS series: adiposederived stromal cells improve the foreign body response. Stem Cells 2008; 26: 2691-5.

18. McFarlane E, Heagy F, Radin S, Aust J, Weemuth E. A study of the delay phenomenon in experimental pedicle flaps. Plast Reconstr Surg 1965; 35: 245-62.

19. Lineaweaver W, Lei M, Mustain W, Oswald T, Cui D, Zhang F. Vascular endothelium growth factor, surgical delay, and skin flap survival. Ann Surg 2004; 239: 866-75.

20. Fraisl P, Mazzone M, Schmidt T, Carmeliet P. Regulation of angiogenesis by oxygen and metabolism. Dev Cell 2009; 16: 167-79.

21. Fraisl P, Aragonés J, Carmeliet P. Inhibition of oxygen sensors as a therapeutic strategy for ischaemic and inflammatory disease. Nat Rev Drug Discov 2009; 8: 139-52.

22. Trojanowska M, Carwile LeRoy E, Eckes B, Krieg T. Pathogenesis of fibrosis: type 1 collagen and the skin. J Mol Med 1998; 76: 266-74.

23. Woodley D, Stanley J, Reese M, O'keefe E. Human dermal fibroblasts synthesize laminin. J Invest Dermatol 1988; 90: 67983.

24. Ceradini D, Kulkarni A, Callaghan M, Tepper O, Bastidas N, Kleinman M, Capla J, Galiano R, Levine J, Gurtner G. Progenitor cell trafficking is regulated by hypoxic gradients through HIF-1 induction of SDF-1. Nat Med 2004; 10: 858-64.

25. Amos P, Shang H, Bailey A, Taylor A, Katz A, Peirce S. IFATS series: the role of human adipose-derived stromal cells in inflammatory microvascular remodeling and evidence of a perivascular phenotype. Stem Cells 2008; 26: 2682-90.

26. Wu Y, Chen L, Scott P, Tredget E. Mesenchymal stem cells enhance wound healing through differentiation and angiogenesis. Stem Cells 2007; 25: 2648-90.

27. Cao Y. Adipose tissue angiogenesis as a therapeutic target for obesity and metabolic diseases. Nat Rev Drug Discov 2010; 9 : 107-15.

28. Dhar SC, Taylor GI. The delay phenomenon: The story unfolds. Plast Reconstr Surg 1999; 104: 2079-91. 